\title{
Magnetic Resonance Imaging-Guided Laser Interstitial Thermal Therapy for Treatment of Drug-Resistant Epilepsy
}

\author{
Joon Y. Kang ${ }^{1}$ (D) $\cdot$ Michael R. Sperling ${ }^{2}$
}

Published online: 30 November 2016

(C) The American Society for Experimental NeuroTherapeutics, Inc. 2016

\begin{abstract}
Surgery is the most effective treatment for drug-resistant epilepsy. Long-term studies demonstrate that about $60 \%$ to $80 \%$ of patients become seizure-free after anterior temporal lobectomy and a majority of patients (about 95\%) report significant seizure reduction after surgery. In the last few years, there has been significant advances in minimally invasive surgical techniques to treat drug-resistant epilepsy. These minimally invasive procedures have significant advantages over open surgery in that they produce less immediate discomfort and disability, while allowing for greater preservation of functional tissue. Laser interstitial thermal therapy (LiTT) is an example of such a procedure. Recent advances in imaging, surgical navigation, and real-time thermal monitoring have made LiTT safer and easier to implement, offering an effective and powerful neurosurgical tool for drug-resistant epilepsy. This article will review the technical considerations, uses, and potential future directions for LiTT in drugresistant epilepsy.
\end{abstract}

Key Words Laser interstitial thermal therapy · drug-resistant epilepsy $\cdot$ epilepsy $\cdot$ temporal lobe epilepsy $\cdot$ mesial temporal sclerosis $\cdot$ temporal lobectomy.

Joon Y. Kang

jkang50@jhmi.edu

1 Johns Hopkins University School of Medicine, 600 N. Wolfe Street, Meyer 2-147, Baltimore, MD 21287, USA

2 Thomas Jefferson University Hospital, 900 Walnut Street Suite 200, Philadelphia, PA 19107, USA

\section{Introduction}

Surgery is the most effective treatment for drug-resistant epilepsy. This is especially true in patients with mesial temporal lobe epilepsy (MTLE). Longitudinal studies show that $60 \%$ to $80 \%$ of patients become seizure-free and $95 \%$ of patients report significant seizure reduction after anterior temporal lobectomy (ATL). Despite these excellent outcomes, epilepsy surgery still remains an underutilized treatment modality. Only about $2 \%$ of the potential surgical candidates annually proceed with epilepsy surgery. The reasons for this underutilization is multifactorial, but, undeniably, one factor for some people is a reluctance to undergo "brain surgery". This stems from concerns about invasiveness of surgery, the risk of permanent neurologic deficits, and pain and discomfort associated with surgery. There are also technical limitations to resective surgery when lesions are deep seated or adjacent to eloquent cortex.

In the last few years, there have been advances in minimally invasive surgical techniques that may be used to treat drug-resistant epilepsy. These minimally invasive procedures have significant advantages over open surgery in that they produce less discomfort and temporary disability, and allow for greater preservation of functional tissue. One example of such a procedure is laser interstitial thermal therapy (LiTT). The principal of thermal ablation to target intracranial lesions is not new: the concept was first utilized in the early 1990s. Recent advances in imaging, surgical navigation, and real-time thermal monitoring have made LiTT safer and easier to implement, offering an effective and powerful neurosurgical tool for drug-resistant epilepsy.

This article will review the technical considerations, uses, and potential future directions for LiTT in refractory epilepsy. 


\section{Principals of LiTT}

LiTT uses high-density, collimated light energy generated by either a continuous wave neodymium-doped yttrium aluminum garndet (Nd: YAG) laser with wavelength $(\lambda=1064 \mathrm{~nm})$ or diode laser $(\lambda=800-980 \mathrm{~nm})$ at various power ranges. Two commonly used commercially available LiTT systems are the Visualase Thermal Therapy System (Medtronics Inc., Fridley, MN, USA) and the NeuroBlate System (Monteris Medical, Inc., Minneapolis, MN, USA). The 2 systems differ in laser catheter design, thermal damage thresholds, and laser type (i.e., the Monteris NeuroBlate System utilizes a 12-W Nd:YAG laser). There is a version of the NeuroBlate System with a rotating, retractable, side-firing probe that can be used for conformed treatment of nongloboid lesions. Most of the clinical discussion for this article is based on experience with the Visualase Thermal system, which uses a 15-W 980-nm diode laser. The diode laser has the advantage of a local peak in water absorption, which creates a sharp thermal boundary between ablated and nonablated tissue [1,2]. The shorter wavelength also allows for shorter penetration depth and avoids damaging the regions outside the target tissue.

During thermal ablation of epileptogenic zone, the diode laser fiber is stereotactically inserted in the target tissue using a magnetic resonance imaging (MRI) compatible applicator with a diffusing tip (see Fig. 1). The diffusing tip creates an ellipsoid light distribution along the axis of the $1-\mathrm{cm}$ tip. When the light energy from the diode laser contacts the tissue, several predictable steps of the laser-tissue interactions occur [3]. The light energy is delivered to cells and converted to heat. Temperatures up to $41{ }^{\circ} \mathrm{C}$ will result in local blood vessel dilation and increased blood flow. At these lower temperatures, the cell responds by initiating a heat shock response, which is a sequence of rapid gene expression to retard thermal induced damage. Cells that survive this initial thermal insult may demonstrate increased thermal resistance. Heating the tissue to temperatures between $42{ }^{\circ} \mathrm{C}$ and $60{ }^{\circ} \mathrm{C}$ will result in denaturation of proteins and DNA, causing irreversible damage. After about $10 \mathrm{~min}$ of exposure in this temperature range, coagulative necrosis occurs. Heating the tissue to temperatures higher than $60{ }^{\circ} \mathrm{C}$ will melt the plasma membrane, denature proteins, and cause instant cell death. An increase of $100{ }^{\circ} \mathrm{C}$ will result in vaporization of water and carbonization. Slight temperature variance in tissue-laser interaction has been reported depending on tissue type, with tumor cells generally more sensitive to heating than normal cells. This is thought be due to variations in oxygen levels and $\mathrm{pH}$ [4]. To our knowledge, temperature sensitivity in different tissue type has yet to be explored in epileptic tissues.

When laser devices were first developed for medical therapy, a major limitation was the inability to detect and monitor the heat delivered to lesions deep in the body. MRI compatibility is one of the major technological advances responsible for recent renaissance of laser thermal therapy in neurosurgery. In 1988, Jolesz et al. [5] were the first to demonstrate that MRI does not interfere with laser-produced thermal therapy and can be used to monitor visually the thermal effect of laser-tissue interaction. Modern systems provide a live "thermal" image of the target tissue and surrounding zones which is refreshed about every $5 \mathrm{~s}$. The software also automatically identifies temperature safety margins so that temperature at the center of the target does not exceed $90^{\circ} \mathrm{C}$, to avoid vaporization and carbonization, and ensures that the temperature of tissue adjacent to the target lesion does not exceed $50{ }^{\circ} \mathrm{C}$. A disposable cooling catheter cools the laser fiber and surrounding tissues to minimize carbonization [6]. Most epilepsy centers set the target cytotoxic temperature between $60{ }^{\circ} \mathrm{C}$ and $80{ }^{\circ} \mathrm{C}$ and multiple ablations (2-3) are performed for selective laser amygdalohippocampectomy [7]. The thermal image is used to approximate the volume ablated or "damage zone" [8].
Fig. 1 Applicator with diffusing tip. During thermal ablation of epileptogenic zone, the diode laser fiber is stereotactically inserted in the target tissue using a magnetic resonance imaging compatible applicator with a diffusing tip. The diffusing tip creates an ellipsoid light distribution along the axis of the $1-\mathrm{cm}$ tip. Image courtesy of Visualase

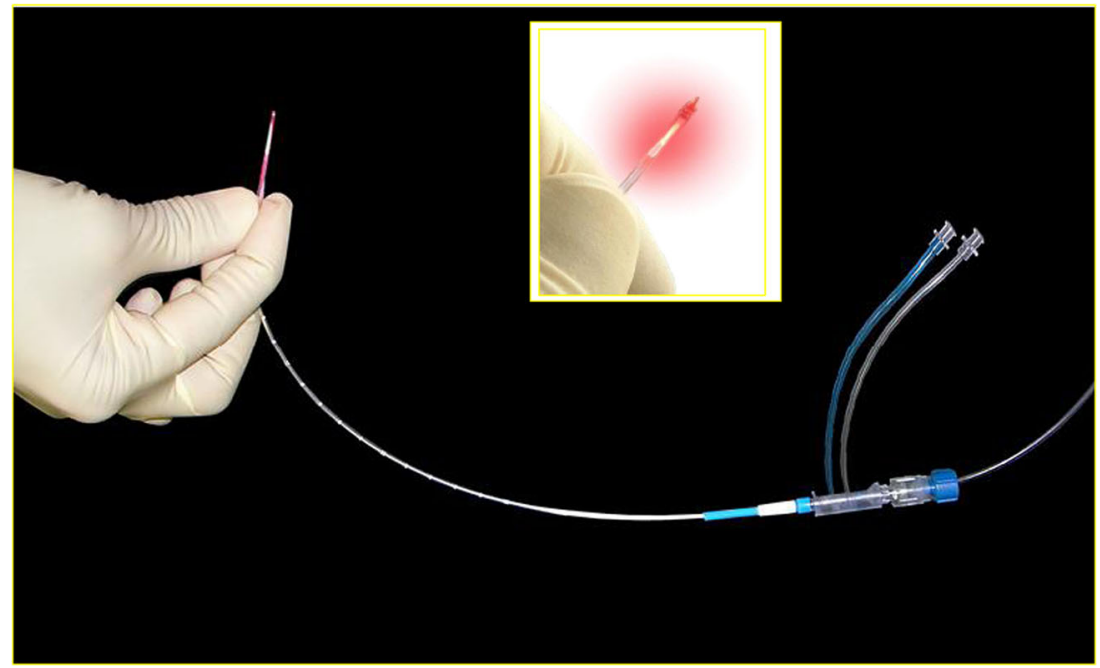




\section{Radiologic-Pathologic Correlation after LiTT}

There is no formal recommendation regarding radiologic follow up after LiTT treatment. Most centers usually perform a baseline T1 contrast-enhanced MRI image on completion of treatment, to assess the volume of ablation (see Fig. 2). Subsequent imaging is performed at the clinician's discretion, depending on the patient's clinical course and seizure response. Much of what is known about LiTT-treated lesions on serial imaging is derived from neuro-oncology therapy. To our knowledge, there is only 1 published review that describes sequential postablation MRI features in patients with nonmalignant lesions [8].

The ablated tissue undergoes changes in the MRI in several stages that correspond to distinct histologically separate concentric zones. Schwabe et al. [9] described 5 concentric zones in 18 patients who underwent LiTT for treatment of brain tumors: 1) light guide track (zone A), 2) central zone (zone B), 3) peripheral zone (zone C), 4) a thin rim at the outer border of the peripheral zone (zone D), and 5) perifocal edema (zone E). The probe track is readily identifiable in the center of the lesion, appearing hypointense on both pre- and postgadolinium magnetic resonance (MR) images and hyperintense on T2-weighted MR images. The probe track diminishes in size over time. The central zone immediately surrounds the probe track and contains blood products, coagulated proteins (i.e. methemoglobin), and necrotic cells. MR images performed within $24 \mathrm{~h}$ may show the central zone to be hyperintense on T1 and hypointense on T2 MRI sequences, owing to high protein levels and heat-induced methemoglobin conversion from deoxyhemoglobin $[9,10]$. In subsequent images, the central zone may appear hypointense on $\mathrm{T} 1$ and

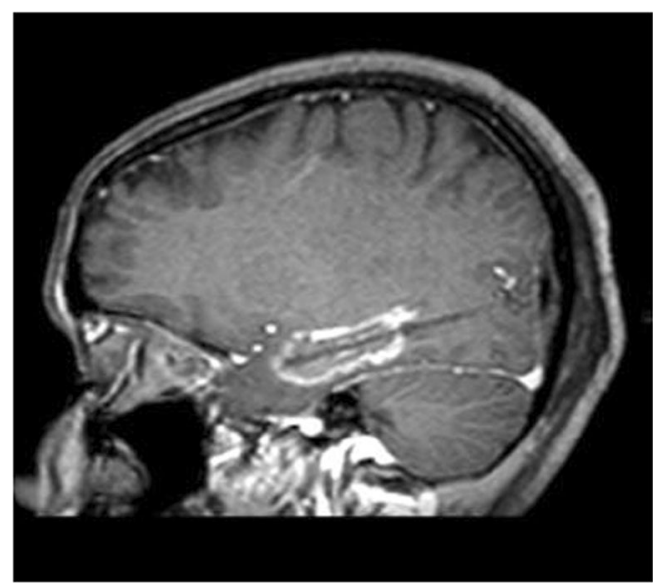

Fig. 2 Postablation magnetic resonance imaging (MRI). This sagittal image shows the ablation cavity immediately at the conclusion of the procedure. A baseline T1 contrast-enhanced MRI image is generally performed on completion of treatment, though this typically overestimates the volume of ablation. It may be repeated several months later, depending upon clinical need hyperintense on T2 MRI sequences depending on the age of blood products and relative concentration of denatured proteins [10]. The peripheral zone contains thrombosed vessels, and shrunken and distended neuronal bodies that undergo delayed liquefaction necrosis [9-11]. The peripheral zone appears hypo- or isointense on $\mathrm{T} 1$ and hyperintense on $\mathrm{T} 2$ MRI sequences $[9,10]$. The peripheral zone increases in size during the first 40 days following the procedure as damaged cells undergo delayed liquefaction necrosis. After 40 days, the size of the peripheral zone gradually decreases [9]. The peripheral contrast-enhancing rim outlines the disrupted bloodbrain barrier and granulation tissue, which on MRI appears as a thin concentric layer of $\mathrm{T} 1$ contrast enhancement around the peripheral zone [9-11]. The diameter of the peripheral rim initially fluctuates with the size of the peripheral zone, or may remain stable through the initial few days, weeks, and months after ablation. The outermost layer is the marginal zone, which contains edematous tissue without thrombosis. This zone initially appears hypointense on T1-weighted MR images and hyperintense on T2-weighted MR images [9]. Edema starts within 1 to 3 days of ablation and reaches a peak after 4 to 27 days (average 6 days) [9]. Regression of edema may take 15 to 45 days (average 23 days) [9].

Atsina et al. [8] reviewed sequential MR images from 23 consecutive patients who underwent LiTT at Thomas Jefferson University Hospital and categorized 4 time periods in which imaging features were clustered. Within hours of surgery (immediate phase), contrast MR images demonstrate a thin peripheral ring of $\mathrm{T} 1$ contrast enhancement (outer zone, zone 3) with a central core consisting of a T1 hyperintense central zone (zone 1) and T1 isointense middle zone (zone 2). As days pass (subacute phase), the edema around the lesion increases, whereas the thin ring of enhancement (outer zone) may stay the same or decrease. Weeks to months after surgery (transitional phase), the central and middle zones are indistinguishable, the ring of contrast enhancement diminish, and edema is nearly resolved. In the chronic phase (months to years following ablation), MRI demonstrates region of gliosis and cavity, in patients with mesial temporal sclerosis (MTS), with little or no contrast enhancement.

\section{Application and Efficacy}

The US Food and Drug Administration first approved the use of an MRI-guided LiTT system for ablation in neurosurgery in 2007. In 2012, Curry and his team at Texas Children's Hospital [12] first reported the feasibility and efficacy of MRI-guided LiTT in ablating epileptic lesions in 5 pediatric patients. Since then, laser has been used to ablate a variety of epileptogenic lesions, including periventricular nodular heterotopia [13], tuberous sclerosis [14], cortical dysplasia 
[12, 14], insular encephalomalacia [15], hypothalamic hamartomas (HH), and MTLE [7, 12, 16, 17]. Although the literature on outcomes is limited to a handful reports, MRguided LiTT is a promising treatment alternative to open resections in patients with MTLE and HH.

\section{MTLE}

Seizure freedom after LiTT in MTLE at 1 to 2 years ranges from $40 \%$ to $60 \%$, with higher rates of seizure freedom seen in patients with MTS on MRI [7, 17]. In 2014, Willie et al. [17] published the results of LiTT therapy in 13 patients who underwent stereotactic laser amygdalohippocampotomy (SLAH) for medically intractable MTLE. About half of their patients were seizure-free after 5 to 26 months. They noted that patients with MTS had slightly higher rates of seizure freedom, but the sample size was too small to form any conclusions. A recent, updated publication by the same group, including the original 13 patients, found similar rates of overall sustained seizure freedom, with 18 of $30(60 \%)$ patients being seizure free at the last follow-up [18]. Most seizure-free patients had MTS (14 of 18) [18]. Similarly, an abstract summarizing results from 7 epilepsy centers presented at the American Epilepsy Society Annual Meeting in 2014 reported that $55 \%$ of patients were seizure-free or had nondisabling simple partial seizures at 1 year after SLAH [19]. Unfortunately, no single standard was applied to define seizure freedom. Patients with MTS had slightly higher rates of seizure freedom than patients without MTS (61\% vs 53\%) at 6 months, but this was not a statistically significant difference. At Thomas Jefferson University Hospital, we observed that about $40 \%$ of the 20 patients who underwent LiTT for MTLE were free of disabling seizures at 2 years [7]. The number of patients as too small for us to compare outcome between patients with and without MTS, but only 1 of the 3 patients without MTS was seizure-free after LiTT. In most cases, seizure recurrence occurred within 6 months of surgery. Patients are typically hospitalized for only 1 day, and most return to work within 1 to 2 weeks of the procedure. Pain medication requirements and length of hospital stay are shorter with LiTT than in patients who undergo ATL [16]. ATL is usually associated with severe postoperative pain, temporary disability for a month or more, and probably has a greater risk of producing complications (the last not yet proven but likely).

Whether seizure-free rates after SLAH are similar to those seen after ATL has yet to be determined. ATL has been reported to results in seizure free rates at 1 to 2 years, ranging from about $60 \%$ to $80 \%$ [20-24]. Seizure-free rates are even higher in patients with MTS $[22,24]$, and about $90 \%$ of the patients report significant improvement in seizure control after ATL. Our own experience suggest that patients usually experience an all-or-none phenomenon after LiTT. Patients who do not become seizure-free often fail to experience substantial reduction in seizure frequency after SLAH.

There is evidence that SLAH may have fewer detrimental effects on neuropsychological performance than ATL. In 2015, Drane et al. [25] compared the pre- and postoperative performance between the group that underwent ATL $(n=39)$ and those that had SLAH $(n=19)$, using the Boston Naming Test and the Iowa Famous Faces test. The Boston Naming Test and the Iowa Famous Faces test assess visual confrontation naming and recognition abilities, respectively. The decline in both measures was significantly greater in patients who underwent ATL compared with patients who had SLAH. None of the patients in the SLAH group (10 dominant, 9 nondominant) exhibited any decline in either of these measures. This is a real advantage, as some postoperative anomia occurs in about $25 \%$ to $65 \%$ of patients after languagedominant hemisphere ATL [26]. SLAH minimizes damage to the anterior lateral temporal cortex, which is critical for naming and recognition from cortical stimulation, functional MRI, and lesion studies [26, 27].

Studies about the effect of SLAH on memory are ongoing, although early results are promising. In our series, we did not observe significant decline in delayed noncontextual and contextual verbal memory after SLAH as assessed by the California verbal learning test and logical memory tests [7]. Decline in verbal memory is associated with dominant hemisphere resections [28]. Sparing lateral temporal cortex may preserve connections involved in contextual learning of information based on semantic content and syntactical structure [29]. However, we expect that ablating mesial temporal lobe structures (hippocampus, parahippocampal gyrus) will result in decline in noncontextual memory, such as learning of unrelated series or pairs of words $[28,29]$. We should note that we have treated patients not yet reported in the literature who did experience verbal memory impairment after SLAH.

The evidence thus far seems to suggest that the SLAH is associated with fewer incidence of major adverse effects compared with open resections. Major postoperative complications after ATL include hemianopia, stroke/hemiparesis, aphasia, and infections (wound, meningitis), with incidence rates ranging from $0.5 \%$ to $4 \%$ [30]. Superior quadrantanopia is probably the most frequent neurologic consequence of ATL, occurring in about one-quarter of patients. Postoperative complications reported after SLAH include visual field deficits (quadrantanopia, homonymous hemianopia), intracranial hemorrhage, cranial nerve palsy, scalp numbness, and worsening mood symptoms $[7,17,19]$. The rate of complication still needs to be determined.

To some extent, the lessons learned from performing intracranial tumor laser ablations may be applied to epilepsy surgery. A recent review by Pruitt et al. [31] highlighted 3 potential points when complications may arise: during catheter placement, LiTT hyperthermia, and technical malfunctioning 
of the cooling system. Suboptimal catheter placement is associated with hemorrhage (subdural, arterial, subarachnoid) at a rate of $1.2 \%$ ( 3 of 243 patients reviewed). Pruitt et al. [31] propose that the risks of hemorrhage may be reduced by using a sharp instrument for dural puncture and fusing computed tomography angiography/MRI and verifying vascular landmark. Commercial systems have safety protocols that shut off the system to prevent tissue vaporization and carbonization so overheating is not a significant concern. Also, blood vessels and the ventricles serve as heat sinks, so the possibility of damage to these structures is remote.

If SLAH turns out to have a lower efficacy rate than ATL, should physicians recommend that patients with MTLE opt for SLAH instead of ATL? The answer may still be "yes" because of advantages related to discomfort, disability, and risk. Moreover, ATL can still be performed should SLAH fail, as was done in several patients in our series who responded well to the second procedure [7].

\section{HH}

$\mathrm{HH}$ are non-neoplastic developmental malformations that are commonly associated with gelastic seizures, although other seizure types (e.g., tonic-clonic seizures) are also seen. Patients also suffer from a constellation of additional symptoms, including behavioral, cognitive, and psychiatric impairments. Prior to MR-guided LiTT, endoscopic and open resections utilizing transcallosal and skull-based approaches resulted in about half of the patients becoming seizure-free after surgery. Unfortunately, open procedures are technically difficult, with high postoperative complication rates, including transient memory impairments, endocrine dysfunction, and cranial nerve palsies including visual field deficits [32]. Alternative methods, such as stereotactic radiosurgery, gamma knife surgery, and radiofrequency ablation are performed but have similar complications as open procedures.

MR-guided LiTT offers a safer approach for the surgical treatment of intractable epilepsy due to $\mathrm{HH}$. The group at Texas Children's Hospital reported the outcome of the largest cases in literature to date, with 14 patients [33]. MR-guided stereotatic laser ablation resulted in 12 of $14(86 \%)$ patients with $\mathrm{HH}$ becoming seizure-free at a mean follow-up of 9 months. Most importantly, there were no permanent surgical complications, including neurologic deficits and endocrine dysfunction. Several other centers have reported similar rates of success without major complications, although reports are limited to case studies [32, 34].

\section{Other Applications}

MR-guided LiTT is well suited for neurosurgical treatment of lesions that are not accessible by open techniques and may be employed as part of a staged approach. A recent case report highlights the potential for an adjunctive role for LiTT in combination with open resection [35]. In this case, a complex frontal lobe cortical dysplasia with extensions deep into the mesial frontal lobe (along the anterior commissure, anterior hypothalamus, and corpus callosum) was initially ablated, then followed with a topectomy of the surgically accessible medial basal frontal lobe guided by electrocorticography (ECoG). The postoperative MRI at 6 months demonstrated no residual cortical dysplasia and the patient was seizurefree at 1 year. LiTT has been also used for disconnection surgeries, including completion of a partial corpus callosotomy with good results [36]. Laser ablation has been used in cavernous malformations $(n=4)$ located in the right frontal lobe, white matter left fusiform gyrus, and right hippocampus with 3 of the 4 patients seizure-free at 6 months [37]. The utility of LiTT extends beyond lesional epilepsy: it has been utilized in nonlesional MTLE [7] and Rasmussen's encephalitis [14] when other methods (e.g., positron emission tomography, intracranial electroencephalography) have identified cortex for ablation. We have also used LiTT to treatment small periventricular and deep white matter nodular heterotopias; the stereotactic approach appears far more comfortable and safer than open resection for these lesions.

\section{Conclusions}

MRI-guided LiTT is a promising alternative to open surgery for some patients with drug-resistant epilepsy, offering better tolerability, reduced incidence of major complications and quicker recovery time. Efficacy needs further assessment, but the greater tolerability and reduced risk warrant use of this method in lieu of open surgery in some patients. Initial reports are promising, but more work is needed to determine optimal ablation volumes and targeting, to improve surgical results. In general, seizure-free rates increase as the volume of resected cortex increases. It is possible that restricted ablation volumes produced by LiTT may not yield similar success rates as open surgery, but the marked diminution in discomfort and lower risk of complication will likely lead to widespread adoption of this technology for treatment of epilepsy, owing to small, readily accessed lesions. Larger, more complex lesions will likely require open surgery, which is also necessary when mapping of eloquent cortex must be done. Tissue can still be obtained for histopathologic examination by stereotactic biopsy prior to performing a thermal ablation, but biopsy volume in these procedures is small and open procedures may be preferred when larger biopsy specimens are needed.

One final comment must be made. Focused ultrasound [38] has been used to ablate small lesions in the brain and elsewhere, and as this technique develops greater targeting 
precision, it is possible that this noninvasive procedure could supplant the minimally invasive LiTT technique.

Required Author Forms Disclosure forms provided by the authors are available with the online version of this article.

\section{References}

1. Jethwa PR, Barrese JC, Gowda A, et al. Magnetic resonance thermometry-guided laser induced thermal therapy for intracranial neoplasms: initial experience. Neurosurgery 2012; 71:133-144.

2. Peavy GM. Laser and laser-tissue interaction. Vet Clinic North Am Small Animal Pract 2002; 32: 517-534.

3. Franck P, Henderson P, Rothaus KO. Basics of lasers history physics and clinical applications. Clin Plast Surg 2016; 43:505-513.

4. Knavel EM, Brace CL. Tumor ablation: common modalities and general practice. Tech Vasc Interv Radiol 2013; 16: 192-200

5. Jolesz FA, Bleier AR, Jakab P, et al. MR imaging of laser-tissue interactions. Radiology 1988;168:249-253

6. Janda P, Sroka R, Mundweil B, et al. Comparison of thermal tissue effects induced by contact application of fiber guided laser systems. Laser Surg Med 2003;33:93-101

7. Kang JY, Wu C, Tracy J, et al. Laser interstitial thermal therapy for medically intractable mesial temporal lobe epilepsy. Epilepsia 2016; 57:325-334.

8. Atsina $\mathrm{KB}$, Sharan $\mathrm{AD}, \mathrm{Wu} \mathrm{C}$, et al. Longitudinal qualitative characterization of MRI features after laser interstitial thermal therapy in drug resistant epilepsy. Am J Roentgenol. 2016; 22:1-9.

9. Schwabe B, Khan T, Harth T, et al. Laser induced thermal lesions in the human brain: short-and long-term appearance on MRI. J Computer Asst Tomogr 1997; 21: 818-825.

10. Medvid R, Ruiz A, Komotor RJ, et al. Current applications of MRIguided laser interstitial thermal therapy in the treatment of brain neoplasms and epilepsy: a radiologic and neurosurgical overview. AJNR Am J Neuroradiol 2015;36: 1998-2006.

11. Schober R, Bettag M, Sabel M, et al. Fine structure of zonal changes in experiemental Nd:YAG laser induced interstitial hyperthermia. Lasers Surg Med 1993:13:234-241.

12. Curry DJ, Gowda A, McNichols RJ, et al. MR-guided stereotactic laser ablation of epileptogenic foci in children. Epilepsy Behav 2012; 24:408-414.

13. Esquenazi Y, Kalamangalam GP, Slater JP, et al. Stereotatic laser ablation of epileptogenic periventricular nodular heterotopia. Epilepsy Res 2014; 108: 547-554.

14. Lewis EC, Wells AG, Duchowny M, et al. MR-guided laser interstitial thermal therapy for pediatric drug resistant lesional epilepsy. Epilepsia 2015;56:1590-1598.

15. Hawasli A, Bandt S, Hogan R, et al. Laser ablation as a treatment strategy for medically refractory dominant insular epilepsy: therapeutic and functional considerations. Stereotact Funct Neurosurg 2014; 92:397-404

16. Waseem H, Osborn KE, Schoenberg M, et al. Laser ablation therapy: an alternative treatment for medically resistant mesial epilepsy after age 50. Epilepsy Behav 2015; 51: 152-157.

17. Willie JT, Laxpati NG, Drane DL, et al. Real-time magnetic resonance guided stereotactic laser amygdalohippocampotomy for mesial temporal lobe epilepsy. Neurosurgery 2014; 74: 569-584.

18. Gross R, Mahmoudi B, Riley J, et al. Less is more: a novel lessinvasive surgical techniques for mesial temporal lobe epilepsy that minimize cognitive impairment. Curr Opin Neurol 2015; 28: 182191.
19. Gross R, Willie J, Helmers S, et al. Stereotatic laser amygdalohippocampotomy for mesial temporal lobe epilepsy: collective experience from seven single-center, prospective, investigator initiated studies. Presented at the American Epilepsy Society Conference; December 2014; Seattle.

20. Engel J (2013) Non-pharamcological therapy of seizures. Seizures and epilepsy (pp. 603-636). New York: Oxford University Press.

21. Wiebe S, Blume WT, Girvin JP, et al. A randomized, controlled trial of surgery for temporal-lobe epilepsy. N Engl J Med 2001;345:311318.

22. Mittal S, Montes JL, Farmer JP, et al. Long-term outcome after surgical treatment of temporal lobe epilepsy in children. J Neurosurg.2005;193:401-412.

23. Kelemen A, Barsi P, Eross L, et al. Long-term outcome after temporal lobe surgery- prediction of late worsening of seizure control. Seizure 2005;15:49-55.

24. Elsharkawy AE, Alabbasi AH, Pannek H, et al. Long-term outcome after temporal lobe epilepsy surgery in 434 consecutive adult patients. J Neurosurg 2009:110:1135-1146.

25. Drane DL, Loring DW, Voets NL et al. Better object recognition and naming outcome with MRI guided stereotactic laser amygdalohippocampotomy for temporal lobe epilepsy. Epilepsia 2015; 56: 101-113.

26. Ives-Deliperi VL, Butler JT. Naming outcome of anterior temporal lobectomy in epilepsy patients: a systematic review of the literature. Epilepsy Behav 2012; 24: 194-198.

27. Abel TJ, Rhome AE, Nourski KV, et al. Direct physiologic evidence of heteromodal convergence region for proper naming in human left anterior temporal lobe. J Neurosci 2015;35: 1513-1520.

28. Tanriverdi T, Dudley R, Hasan A, et al. Memory outcome after temporal lobe epilepsy surgery: corticoamygdalohippocampectomy versus selective amygdalohippocampectomy. J Neurosurg 2010; 113: 1164-1175.

29. Saling MM. Verbal memory in mesial temporal lobe epilepsy: beyond material specificity. Brain 2009; 132:570-582.

30. Attiah M, Paulo D, Danish S, et al. Anterior temporal lobectomy compared with laser thermal hippocampectomy for mesial temporal epilepsy: a threshold analysis. Epilepsy Res 2015; 115:1-7.

31. Pruitt R, Gambel A, Black K, et al. Complication avoidance in laser interstitial thermal therapy lessons learned. J Neurosurg 2016; 3: 18.

32. Rolston JD, Chang EF. Stereotactic laser ablation for hypothalamic hamartoma. Neurosurg Clin North Am 2016; 27: 59-67.

33. Wilfong A, Curry DJ. Hypothalamic hamartomas: optimal approach to clinical evaluation and diagnosis. Epilepsia 2013; 54: 109-114.

34. Brandmeir N, Achara V, Sather M. Robot assisted stereotactic laser ablation for radiosurgery resistant hypothalamic hamartoma. Cureus 2016; 8: 6-10

35. Ellis JA, Mejia Munne JC, Wang SH, et al. Staged laser interstitial thermal therapy and topectomy for complete obliteration for focal cortical dysplasia. J Clin Neurosci 2016; 31: 224-228.

36. Ho A, Miller KJ, Cartmell S et al. Stereotactic laser ablation of the splenium for intractable epilepsy. Epilepsy Behav Case Rep 2016; 5: 23-26.

37. Drane D, Willie J, Loring D, et al. Cognitive outcome of patients undergoing stereotactic laser ablation of cavernous malformation to control chronic seizures. Presented at the American Epilepsy Society Conference; December 2014; Seattle.

38. Monteith S, Snell J, Eames M, et al. Transcranial magnetic resonance-guided focused ultrasound for temporal lobe epilepsy: a laboratory feasibility study. J Neurosurg 2016; 12: 1-8. 